\title{
Sur la spécialisation des points extrémaux cubiques
}

par

\section{R. Paysant-Le Roux, M. L. Gaunet et E. Dubois (Caen)}

I. Introduction. L'algorithme des fractions continues intervient dans beaucoup de domaines de la théorie des nombres. Dans un corps de séries de Laurent $Q((1 / X))$ on peut aussi considérer l'algorithme des fractions continues, dites formelles, en partant de la relation

$$
\alpha=\left(a_{-d} X^{d}+\ldots+a_{0}\right)+\left(\frac{a_{1}}{X}+\ldots\right)=E(\alpha)+F(\alpha)
$$

avec

$$
E(\alpha) \in Q[X], \quad F(\alpha) \in Q[[1 / X]] .
$$

L'étude des liens entre les réduites du développement en fraction continue formelle que l'on spécialise (remplacer l'indéterminée $X$ par un entier $n$ ) et les réduites du développement en fraction continue réelle du même nombre spécialisé ont conduit plusieurs auteurs $[5,3,4,1]$ à des résultats sur les réels.

Dans une autre direction, l'algorithme des fractions continues ne se prolonge pas canoniquement à plusieurs nombres réels. Depuis une dizaine d'années, plusieurs auteurs (E. Dubois, J. Brentjes, H. Appelgate et H. Onishi, J. Buchmann) ont considéré l'aspect meilleure approximation des réduites du développement en fractions continues d'un nombre réel et ont introduit de nouvelles notions de meilleure approximation qui ont permis d'obtenir des algorithmes, généralisant celui des fractions continues à plusieurs nombres réels, ayant de bonnes propriétés du point de vue des approximations rationnelles simultanées de deux ou plusieurs nombres réels, approximations par une forme linéaire, et du point de vue du calcul d'unités. Dans un corps de nombres algébriques, on peut mettre une structure de graphe sur l'ensemble des meilleures approximations d'un ordre de ce corps. Le quotient de ce graphe par les unités de cet ordre est alors fini. Son cardinal est appelé calibre de cet ordre. $D$ ans le cas d'un corps quadratique réel, ce graphe quotient est en bijection avec la période primitive du développement en fraction continue. Pour $P(X) \in Z[X]$ de degré $3 d$ nous étudions le calibre de $Z[\sqrt[3]{P(n)}]$ lorsque $n$ décrit les entiers.

Le résultat principal de ce papier est de montrer que, dans le cas d'un polynôme unitaire de degré $3 d$, on peut partitionner l'ensemble des entiers 
en au plus deux classes telles que dans l'une le calibre de l'ordre $Z[\sqrt[3]{P(n)}]$ est borné indépendamment de $n$ et dans la seconde le calibre tend vers l'infini avec $n$.

A cette fin, une méthode est d'introduire et d'étudier des meilleures approximations formelles que nous appellerons points extrémaux. L'un des auteurs [4] a déjà considéré ces objets pour des extensions cubiques pures de $Q(X)$ contenues dans $Q((1 / X))$. Il en déduisait déjà des conséquences dans le cas réel. Citons le résultat suivant:

Si pour tout $n$ assez grand l'unité de $Q\left(\sqrt[3]{n^{3}+a n+b}\right)$ a une forme polynomiale en $n$ (c'est-à-dire de la forme $u_{0}(n)+u_{1}(n)\left(n^{3}+a n+b\right)^{1 / 3}+u_{2}(n)\left(n^{3}+a n+b\right)^{2 / 3}$ avec $\left.u_{j}(n) \in \boldsymbol{Z}[n]\right)$ alors nécessairement $a=0$ ou $b=0$ ou $(a, b) \in\left\{\left(-6 h^{2}, 6 h^{3}\right)\right.$, $\left.\left(-9 h^{2}, 9 h^{3}\right), h \in \boldsymbol{Z}\right\}$.

Nous nous proposons d'étudier l'interface entre le cas réel et le cas formel.

\section{Points extrémaux.}

(a) Meilleure approximation. Dans un corps cubique pur de la forme $K=\boldsymbol{Q}(\sqrt[3]{D}) \subset \boldsymbol{R}$ avec $D \in \boldsymbol{Z}^{*}$ et $D$ non cube parfait dans $\boldsymbol{Z}$, on désigne par $|\cdot|_{1}$ et $|\cdot|_{2}$ les deux valeurs absolues archimédiennes normalisées définies par

$$
|\varphi|_{1}=|\varphi|, \quad|\varphi|_{2}=\left|\varphi^{\prime}\right|^{2}
$$

si $\varphi$ est un élément de $K$ et si $\varphi^{\prime}$ désigne un conjugué de $\varphi$.

Nous généralisons la notion de réduite du développement en fraction continue d'un nombre réel quadratique par celle de meilleure approximation relative à un ordre $\mathcal{O}$ de $K$.

DÉFINITION 1. $\varphi \in \mathcal{O}-\{0\}$ est une meilleure approximation de l'ordre $\mathcal{O}$ si pour tout $\psi \in \mathcal{O}-\{0\}$ vérifiant $|\psi|_{i} \leqslant|\varphi|_{i}$ pour $i=1,2$ on a $\psi= \pm \varphi$.

On définit également la notion de deux meilleures approximations voisines.

DÉFINITION 2. Deux meilleures approximations $\varphi$ et $\delta$ sont dites voisines si pour tout $\psi \in \mathcal{O}$ vérifiant $|\psi|_{i}<\operatorname{Max}\left\{|\varphi|_{i},|\delta|_{i}\right\}$ pour $i=1,2$ on a $\psi=0$.

Une meilleure approximation $\varphi$ (modulo \pm 1 ) a exactement deux meilleures approximations voisines $\delta, \eta$ telles que

$$
|\delta|_{1}<|\varphi|_{1}<|\eta|_{1}, \quad|\delta|_{2}>|\varphi|_{2}>|\eta|_{2} .
$$

En remarquant que 1 est une meilleure approximation, on obtient ainsi une double chaîne infinie de meilleures approximations
On désigne par $\mathscr{E}$ l'ensemble des meilleures approximations de $\mathcal{O}$. En partant de $\varphi^{(0)}=1$, ces chaînes contiennent les unités $\mathscr{U}$ de $\mathcal{O}$ et la première unité rencontrée est l'unité fondamentale de $\mathcal{O}$. On remarque de plus que $\mathscr{U}$ opère sur $\mathscr{E}$, ce qui permet de considérer l'ensemble quotient $\mathscr{E} / \mathscr{U}$. Celui-ci est fini. La structure de graphe de $\mathscr{E}$ passe aussi au quotient, on obtient un cycle:

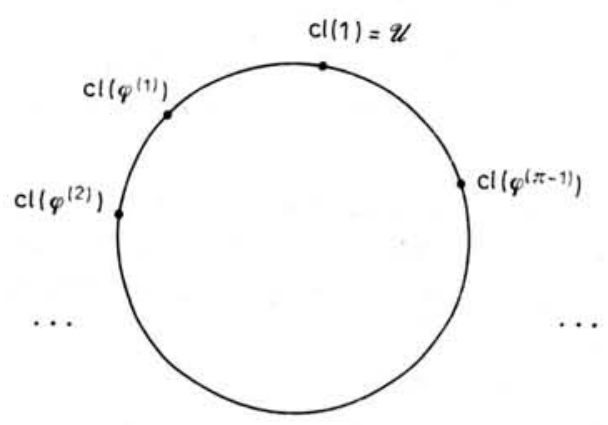

Dans le cas quadratique réel se donner le graphe quotient de l'ordre $\mathcal{O}=\boldsymbol{Z}[\sqrt{D}]$ avec $D>0$ non carré est équivalent à se donner la période du développement en fraction continue de $\sqrt{D}$, en particulier le nombre d'éléments du cycle est égal à la longueur de la période primitive.

(b) Point extrémal formel. On se donne maintenant un polynôme $P(X)$ à coefficients entiers, unitaire de degré $3 d$ avec $d$ entier supérieur ou égal à 1 , non cube dans $Q[X]$ :

$$
P(X)=X^{3 d}+a_{1} X^{3 d-1}+\ldots+a_{3 d} \in \mathbf{Z}[X],
$$

et nous considérons le corps de fonctions $K_{X}=Q(X, W)$, où $W=X^{d}+\ldots$ est la racine dans $Q((1 / X))$ de l'équation $Y^{3}=P(X)$, et les meilleures approximations de l'ordre $\mathcal{O}_{X}=Q[X, W]$ que nous appellerons ici points extrémaux.

Le corps de fonctions $K_{X}$ est une extension cubique de $Q(X)$ qui possède deux valeurs absolues $|\cdot|_{1}$ et $|\cdot|_{2}$ au dessus de la valeur absolue à l'infini de $Q(X)$ définie par $|X|=e$ avec $e>1$ et triviale sur $Q$. Plus précisément, si $\varphi \in K_{X}$, on a

$$
\varphi=P(X)+Q(X) W+R(X) W^{2}=c_{-t} X^{-t}+c_{t-1} X^{-t-1}+\ldots \in Q((1 / X)),
$$

avec $c_{-t} \neq 0$, et si $\varphi^{\prime}$ désigne le conjugué de $\varphi$,

$$
P+Q \zeta W+R \zeta^{2} W^{2}=d_{q} X^{q}+d_{q-1} X^{q-1}+\ldots \in Q(\zeta)((1 / X)),
$$

avec $d_{q} \neq 0$, on pose

$$
|\varphi|_{1}=e^{-t} \quad \text { et } \quad|\varphi|_{2}=e^{2 q} .
$$

de $\hat{O}_{x}$. 
DéFINITION 1'. $\varphi \in \mathcal{O}_{X}-\{0\}$ est un point extrémal de l'ordre $\mathcal{O}_{X}$ si pour tout $\psi \in \mathcal{O}_{X}-\{0\}$ vérifiant $|\psi|_{i} \leqslant|\varphi|_{i}$ pour $i=1,2$ on a $\psi=\lambda \varphi$ avec $\lambda \in Q^{*}$ (i.e. $|\psi|_{i}=|\varphi|_{i}$ pour $i=1$ et 2 ).

Si nous demandons simplement, dans la définition 1', qu'il existe un $i$ tel que $|\psi|_{i}=|\varphi|_{i}$, nous avons ce que nous appellerons une face. Elles interviennent de façon naturelle dans notre problème.

DÉfinition 3. $\varphi \in \mathcal{O}-\{0\}$ est une face de l'ordre $\mathcal{O}_{X}$ si pour tout $\psi \in \mathcal{O}_{X}-\{0\}$ vérifiant $|\psi|_{i} \leqslant|\varphi|_{i}$ pour $i=1,2$ il existe au moins un $i$ tel que $|\psi|_{i}=|\varphi|_{i}$.

On note par $\mathscr{E}_{X}$ (resp. $\mathscr{F}_{X}$ ) l'ensemble des points extrémaux (resp. faces) de $\mathcal{O}_{X}$ définis à une constante rationnelle non nulle multiplicative près.

Enfin notons par $\mathscr{U}_{X}$ (resp. $\mathscr{U}_{X}^{(1)}$ ) le groupe des unités de l'ordre $\mathcal{O}_{X}$ (resp. des unités de norme 1).

On a les injections suivantes:

$$
\mathscr{U}_{X}^{(1)} \subset \mathscr{U}_{X} / \boldsymbol{Q}^{*} \subset \mathscr{E}_{X} \subset \mathscr{F}_{X} .
$$

Les groupes $\mathscr{U}_{X} / \boldsymbol{Q}^{*}$ et $\mathscr{U}_{X}^{(1)}$ opèrent sur $\mathscr{E}_{X}$ (resp. $\mathscr{F}_{X}$ ). La notion de deux points extrémaux voisins est utile pour mettre une structure de graphe sur $\mathscr{E}_{X}$.

DéFINITION $2^{\prime}$. Deux points extrémaux $\varphi$ et $\delta$ sont dits voisins si pour tout $\psi \in \mathcal{O}$ vérifiant $|\psi|_{i}<\operatorname{Max}\left\{|\varphi|_{i},|\delta|_{i}\right\}$ pour $i=1,2$ on a $\psi=0$.

La proposition suivante est facile à montrer.

Proposition 1. L'ensemble $\mathscr{E}_{X}$ des points extrémaux, modulo $Q^{*}$, est ordonnée par $|\varphi|_{2}$ croissante et forme alors une suite $\left(\varphi_{k}\right)_{k \in \mathbf{Z}}$. Deux points extrémaux sont voisins si et seulement si ils sont consécutifs dans la suite $\left(\varphi_{k}\right)_{k \in \mathbf{Z}}$.

$\mathscr{E}_{X}$ a donc comme structure de graphe une double chaîne infinie. Cette structure passe au quotient par $\mathscr{U}_{X}^{(1)}$ et par $\mathscr{U}_{X} / Q^{*}$ et on a

Proposition 2. Les graphes quotients de $\mathscr{E}_{X}$ par $\mathscr{U}_{X}^{(1)}$ et par $\mathscr{U}_{X} / \mathbf{Q}^{*}$ sont finis si et seulement si $\mathscr{U}_{X} \neq Q^{*}$, c'est-à-dire si le groupe $\mathscr{U}_{X}$ est non trivial.

En effet, si ces graphes quotients sont finis alors nécessairement il existe une unité non triviale, $\varepsilon$, dans $\mathscr{U}_{X}$ et donc dans $\mathscr{U}_{X}^{(1)}$ en considérant $\varepsilon^{3} / N(\varepsilon)$.

Réciproquement, s'il existe une unité non triviale dans $\mathscr{U}_{X}$ alors le groupe $\mathscr{U}_{X} / Q^{*}$ est d'après [2] de rang 1 .

D'autre part, il est facile de montrer que:

- toute unité $\varepsilon$ de $\mathscr{U}_{X}$ est un point extrémal de $\mathcal{O}_{X}$,

- si $\varphi \in \mathscr{E}_{X}$ et si $\varepsilon=\mathscr{U}_{X}$ alors $\varepsilon \varphi \in \mathscr{E}_{X}$,

- si $\varphi$ et $\psi$ sont deux points extrémaux voisins et si $\varepsilon \in \mathscr{U}_{X}$ alors $\varepsilon \varphi$ et $\varepsilon \psi$ sont voisins. finis. (c) Calcul des points extrémaux. Le calcul effectif d'un point extrémal de la suite $\left(\varphi_{k}\right)_{k} \geqslant 0$ peut se faire en résolvant des systèmes linéaires en des inconnues rationnelles. Soit $q$ un entier naturel et cherchons un point extrémal avec $U_{i} \in Q[X], \quad 0 \leqslant i \leqslant 2$, et $\operatorname{Max}\left(\operatorname{deg}\left(U_{0}\right), \operatorname{deg}\left(U_{1} \Delta\right), \operatorname{deg}\left(U_{2} \Delta^{2}\right)\right) \leqslant q$, c'est-à-dire en notant $\varphi=U_{0}+U_{1} \Delta+U_{2} \Delta^{2}$, avec $|\varphi|_{2} \leqslant e^{2 q}$.

Il suffit de construire $\varphi$ tel que $|\varphi|_{1}$ soit minimal. Il faut donc, dans la série de Laurent en $1 / X$ de $\varphi(X)$, annuler le maximum de coefficients parmi les termes de plus grand degré en $X$.

Les coefficients inconnus de $U_{0}, U_{1}, U_{2}$ sont au nombre de $3(q-d+1)$ dès que $q \geqslant 2 d$. (Si $q<d, U_{1}=U_{2}=0$ et $\varphi=1$ est le seul point extrémal. $\mathrm{Si}$ $d \leqslant q<2 d, U_{2}=0$ et il y a $2 q+2-d$ inconnues).

Plaçons-nous dans le cas principal $q \geqslant 2 d$.

Si l'on considère $3(q-d)+2$ équations, celles correspondant à l'annulation des termes de degré $q, q-1, \ldots,-(2 q-3 d+1)$ dans $\varphi(X)$, il y a une solution non triviale. Il existe donc une solution $\varphi \in \mathcal{O}_{X} \backslash\{0\}$ avec $|\varphi|_{1} \leqslant e^{-(2 q-3 d+2)}$. Soit $t$ maximum tel qu'il existe une solution $\varphi \in \mathcal{O}_{X} \backslash\{0\}$ non triviale vérifiant $|\varphi|_{1}=e^{-t}$ et $|\varphi|_{2} \leqslant e^{2 q}$. On a donc $t \geqslant 2 q-3 d+2$. D'autre part, la norme de $\varphi=\varphi \varphi^{\prime} \varphi^{\prime \prime}$ est un polynôme de degré positif ou nul. On a donc $|\varphi|_{1} e^{2 q}$ $\geqslant|\varphi|_{1}|\varphi|_{2} \geqslant 1$ et $-t+2 q \geqslant 0$.

Dans le cas $d \leqslant q<2 d$ on obtient par le même raisonnement $q-d+1 \leqslant t \leqslant 2 q$.

Montrons que $\varphi$ ainsi défini est un point extrémal et est unique.

Soit $\psi \in \mathcal{O}_{X} \backslash\{0\}$ tel que $|\psi|_{i} \leqslant|\varphi|_{i}, i=1,2$. De $t$ maximum on déduit $|\psi|_{1}=|\varphi|_{1}$. Il existe donc un rationnel $\lambda$ tel que $|\varphi-\lambda \psi|_{1}<e^{-t}$. D'autre part, $|\varphi-\lambda \psi|_{2} \leqslant e^{2 q}$ et puisque $t$ est maximum on a $\varphi=\lambda \psi$. Ceci prouve aussi l'unicité a une constante rationnelle non nulle près de $\varphi$.

$\mathrm{Si}$ on pose $|\varphi|_{2}=e^{2 q^{\prime}}$, où $\varphi$ est la fonction construite ci-dessus, on en déduit la proposition suivante:

Proposition 3. Pour tout $q \geqslant 0$, il existe $q^{\prime} \leqslant q$ et un point extrémal $\varphi$ vérifiant $|\varphi|_{2}=e^{2 q^{\prime}}$ tels que en notant $|\varphi|_{1}=e^{-t}$ on ait

$$
\begin{cases}q^{\prime}=0, t=0 & \text { pour } 0 \leqslant q<d, \\ q-d+1 \leqslant t \leqslant 2 q^{\prime} & \text { pour } d \leqslant q<2 d, \\ 2 q-3 d+2 \leqslant t \leqslant 2 q^{\prime} & \text { pour } 2 d \leqslant q .\end{cases}
$$

Remarque. La fonction $\varphi$ de la proposition 3 est une unité si et Seulement si $t=2 q^{\prime}$, et dans le cas $d=1$ on a toujours $q^{\prime}=q$ et $t$ ne peut prendre que les valeurs $2 q$ ou $2 q-1$.

Pour faciliter la rédaction, nous introduisons des notations matricielles en se plaçant dans le cas $q \geqslant 2 d$. Les modifications à apporter pour $d \leqslant q<2 d$ sont faciles à écrire. En écrivant

$$
\Delta=\sum_{i=d}^{-\infty} a_{i}^{(1)} X^{i} \quad\left(a_{d}^{(1)}=1\right) \quad \text { et } \quad \Delta^{2}=\sum_{i=2 d}^{-\infty} a_{i}^{(2)} X^{i} \quad\left(a_{2 d}^{(2)}=1\right)
$$


on considère les matrices $A_{q, \infty}$ formées des blocs $A_{q, \infty}^{(j)}, j=0,1,2$, qui ont respectivement $q+1, q-d+1, q-2 d+1$ colonnes et une infinité de lignes. La ligne, notée $-k$, de la matrice $A_{q, \infty}$ correspond aux termes des séries en $1 / X$ représentant $1, \Delta, \Delta^{2}$ qui interviennent dans le terme de degré $-k$ de la série $U_{0}+U_{1} \Delta+U_{2} \Delta^{2}$.

$$
\begin{aligned}
& \text { ligne } q \quad 1,0, \ldots 0, \quad 1, \quad 0, \ldots \quad 0, \quad 1, \quad 0, \ldots 0 \\
& \text { ligne } q-1 \quad 0,1, \ldots 0, \quad a_{d-1}^{(1)}, \quad 1, \ldots \quad 0, \quad a_{2 d-1}^{(2)}, \quad 1, \ldots 0 \\
& \text { ligne } 0 \quad 0,0, \ldots 1, \quad a_{d-q}^{(1)}, \quad a_{d-q+1}^{(1)} \ldots a_{0}^{(1)}, \quad a_{2 d-q}^{(2)}, \quad 1, \ldots a_{0}^{(2)} \\
& \text { ligne }-k \quad 0,0, \ldots 0, \quad a_{d-q-k}^{(1)}, \ldots \quad a_{-k}^{(1)}, \quad a_{2 d-q-k}^{(2)}, \ldots a_{-k}^{(2)} \\
& \ldots \ldots \ldots \ldots \ldots \ldots \ldots \ldots \ldots \ldots \ldots \ldots \ldots \ldots \ldots \ldots \ldots \\
& A_{q . \infty}^{(0)} \\
& A_{q, \infty}^{(1)}
\end{aligned}
$$

On notera $A_{q, m}$ la matrice formée des $m$ premières lignes de $A_{q, \infty}$, c'est-à-dire des lignes $q, q-1, \ldots, q-m+1$.

En posant $U_{j}(X)=u_{q-j d}^{(j)} X^{q-j d}+\ldots+u_{\delta}^{(j)}$ pour $j=0,1,2$ et $U^{(q)}$ le vecteur colonne ${ }^{t}\left(u_{q}^{(0)}, \ldots, u_{0}^{(0)}, u_{q-d}^{(1)}, \ldots, u_{0}^{(1)}, u_{q-2 d}^{(2)}, \ldots, u_{0}^{(2)}\right)$, le produit matriciel $A_{q, \infty} U^{(q)}$ est composé dẹ coefficients de la série de Laurent $U_{0}(X)+U_{1}(X) \Delta+U_{2}(X) \Delta^{2}$ alors que $A_{q, m} U^{(q)}$ est composé des premiers coefficients de cette série. Avec ces notations le $t$ (dépendant de $q$ ) défini à la proposition 3 est la valeur maximale de $m-q$ tel que l'équation $A_{q, m} U^{(q)}=0$ ait une solution $U^{(q)}$ rationnelle non nulle. Pour cet $U^{(q)}, A_{q, m+1} U^{(q)} \neq 0$. On peut énoncer

Proposition 4. (i) Si $\varphi$ est un point extrémal vérifiant $|\varphi|_{1}=e^{-t}$ avec $t>0,|\varphi|_{2}=e^{2 q}$ on $a$

$$
\operatorname{rang}\left(A_{q, q+t+1}\right)=1+\operatorname{rang}\left(A_{q, q+t}\right)=3(q-d+1) .
$$

(ii) $S i$ (2) est satisfaite, il existe $q^{\prime} \leqslant q$ et un point extrémal $\varphi$ vérifiant $|\varphi|_{1}=e^{-t},|\varphi|_{2}=e^{2 q^{\prime}}$ et pour tout $q^{\prime \prime}, q^{\prime} \leqslant q^{\prime \prime} \leqslant q$, on a

$$
\operatorname{rang}\left(A_{q^{\prime \prime}, q^{\prime \prime}+t+1}\right)=1+\operatorname{rang}\left(A_{q^{\prime \prime}, q^{\prime \prime}+t}\right)=3\left(q^{\prime \prime}-d+1\right)
$$

et $2\left(q-q^{\prime}\right) \leqslant 3 d-2$.

(iii) $\operatorname{Si} \varphi$ et $\pi$ sont des points extrémaux voisins tels que $|\varphi|_{1}=e^{-t}$, $|\varphi|_{2}=e^{2 q_{1}},|\pi|_{1}=e^{-t_{2}},|\pi|_{1}=e^{2 q_{2}}$ avec $t_{1}<t_{2}, q_{1}<q_{2}$ alors pour tout $q$ tel que $q_{1} \leqslant q<q_{2}$ on $a$

$$
\operatorname{rang}\left(A_{q . q+t_{1}}+1\right)=1+\operatorname{rang}\left(A_{q . q+t_{1}}\right)=3(q-d+1) .
$$

Preuve. (i) Soit $\varphi$ un point extrémal tel que $|\varphi|_{1}=e^{-t},|\varphi|_{2}=e^{2 q}$. Alors pour tous $V_{0}, V_{1}, V_{2}$ dans $\boldsymbol{Z}[X]$ de degrés respectifs majorés par $q, q-d, q-2 d$ on a $\left|V_{0}+V_{1} \Delta+V_{2} \Delta^{2}\right|_{1} \geqslant e^{-t}$ et donc $A_{q, q+t+1} V^{(q)} \neq 0$ si $V^{(q)}$ est le vecteur composé des coefficients de $V_{0}, V_{1}, V_{2}$. D'autre part, $\varphi$ donne une solution $U^{(q)}$ de $A_{q, q+1} U^{(q)}=0$. Alors (2) en résulte facilement puisque d'après (1) le nombre de lignes, $q+t+1$, est supérieur ou égal au nombre de colonnes, $3(q-d+1)$, de $A_{q \cdot q+t+1}$.

(ii) Réciproquement, soient $t$ et $q$ tels que (2). Puisque $\operatorname{rang}\left(A_{q, q+t}\right)$ $<3(q-d+1)$ il existe une solution non triviale $U$, dans $Q^{(3 q-d+1)}, \mathrm{de}$ $A_{q, q+t} U=0$. Comme $\operatorname{rang}\left(A_{q, q+t+1}\right)=3(q-d+1)$ on a $A_{q, q+t+1} U \neq 0$. Avec les composantes de $U$ on forme les polynômes $U_{0}, U_{1}, U_{2}$ tel que le vecteur $U^{(q)}$ défini précédemment soit $U$. Alors $\varphi=U_{0}+U_{1} \Delta+U_{2} \Delta^{2}$ vérifie $|\varphi|_{1}=e^{-t}$. Soit $q^{\prime}$ défini par $|\varphi|_{2}=e^{2 q^{\prime}}$. On a $q^{\prime} \leqslant q$.

Montrons que $\varphi$ est un point extrémal. Soit $\psi=V_{0}+V_{1} \Delta+V_{2} \Delta^{2} \neq 0$ tel que $|\psi|_{i} \leqslant|\varphi|_{i}$ pour $i=1,2$. Avec les coefficients des polynômes $V_{0}, V_{1}, V_{2}$ formons un élément $V^{(q)}$ de $Q^{3(q-d+1)}$ en complétant éventuellement par des 0 . On a alors $A_{q, q+t} V^{(q)}=0$, mais comme $\operatorname{dim} \operatorname{ker}\left(A_{q, q+t}\right)=1$ on a $V^{(q)}=\lambda u^{(q)}$ avec $\lambda \in Q^{*}$, soit $\psi=\lambda \varphi$. $\varphi$ est donc un point extrémal.

D'après (i), (2) est satisfaite en remplaçant $q$ par $q^{\prime}$. Et comme le passage de $q^{\prime \prime}$ à $q^{\prime \prime}+1$ augmente le rang des matrices d'au plus 3 (le nombre de colonnes en plus) on a (2) pour tout $q^{\prime \prime}$ entre $q^{\prime}$ et $q$.

Enfin, d'après (1) on a $2 q-3 d+2 \leqslant 2 q^{\prime}$ et donc $2\left(q-q^{\prime}\right) \leqslant 3 d-2$. On retrouve la remarque qui suit la proposition 3: pour $d=1$ il existe pour tout $q$ un point extrémal $\varphi$ tel que $|\varphi|_{2}=e^{2 q}$ car on a toujours $q=q^{\prime}$.

(iii) résulte immédiatement de (ii).

III. Spécialisation des points extrémaux. Dans ce paragraphe on se donne un polynôme $P(X)=X^{3 d}+\ldots+a_{3 d}$, à valeurs entières sur $\boldsymbol{Z}$, non cube. On note par $\tilde{Z}$ l'ensemble des entiers tels que $P(n)$ ne soit pas un cube dans $\boldsymbol{Z}$ et par $\mathcal{O}_{n}$ l'ordre $Z[\sqrt[3]{P(n)}]$. On considère la racine $\Delta=X^{d}+\ldots$ dans $Q((1 / X))$ de $\Delta^{3}=P(X)$ et l'ordre $\mathcal{O}_{X}=Q[X, \Delta]$.

Pour qu'un point extrémal $\varphi=U_{0}(X)+U_{1}(X) \Delta+U_{2}(X) \Delta^{2}$ donne par spécialisation une meilleure approximation $\varphi(n)$, il faut d'abord rendre $U_{0}(n), U_{1}(n), U_{2}(n)$ entiers, premiers dans leur ensemble.

Proposition 5. Soit $\varphi$ un point extrémal de $\mathcal{O}_{X}$. Alors il existe un nombre fini $s$ de couples $\left(E_{i}, \varphi^{(i)}\right), i=1, \ldots, s$, tels que:

(i) les sous-ensembles $E_{i}$ forment une partition de $\tilde{Z}$,

(ii) pour tout $i$ dans $[1, s], \varphi^{(i)}$ est une fonction algébrique $U_{0}^{(i)}(X)$ $+U_{1}^{(i)}(X) \Delta+U_{2}^{(i)}(X) \Delta^{2}$, équivalente à $\varphi$ (i.e. $\left.\exists \lambda \in Q^{*}, \varphi^{(i)}=\lambda \varphi\right)$, telle que $U_{0}^{(i)}(n), U_{1}^{(i)}(n), U_{2}^{(i)}(n)$ sont, pour tout $n$ dans $E_{i}$, des entiers premiers dans leur ensemble.

Preuve. Soit $\varphi$ un point extrémal formel de $\mathcal{O}_{x} ;$ comme il est défini à une constante multiplicative non nulle près, on peut l'écrire $\varphi=U_{0}+U_{1} \Delta+U_{2} \Delta^{2}$ avec $U_{i} \in Z[X]$ pour $i=0,1,2$.

D'autre part, $\varphi$ étant un point extrémal, le plus grand commun diviseur dans $Q[X]$ de $\left(U_{0}, U_{1}, U_{2}\right)$ est une constante non nulle de $Q$. 
On en déduit

$\exists d \in N^{*}, \quad \exists v_{0}, v_{1}, v_{2} \in \boldsymbol{Z}[X]$ tels que $v_{0} U_{0}+v_{1} U_{1}+v_{2} U_{2}=d ;$

on peut supposer $d$ minimum.

Soient $p$ un nombre premier, $k$ un entier naturel et $n$ dans $\tilde{Z}$. D'après la relation précédente le système de congruences $U_{j}(n) \equiv 0\left[\bmod p^{k}\right], j=0,1,2$, ne peut être vèrifié que si $p^{k}$ divise $d$. La proposition 5 en résulte.

EXEMPLE. Si $\Delta^{3}=X^{3}-9 X+9,\left(X^{3} / 3\right)-3 X+4+\left(-\left(X^{2} / 3\right)-X+2\right) \Delta+\Delta^{2}$ est un point extrémal de $\mathcal{O}_{X}$ [4]. Posant $U_{0}=X^{3}-9 X+12, U_{1}=-X^{2}$ $-3 X+6, U_{2}=3$, on a

$$
(X+4) U_{0}+\left(X^{2}+X-6\right) U_{1}-3 U_{2}=3 .
$$

On en déduit la partition de $\tilde{Z}=Z$ et les fonctions $\varphi^{(i)}$ :

$$
E_{1}=3 Z, \quad \varphi^{(1)}=\varphi(n) ; \quad E_{2}=Z \backslash E_{1}, \quad \varphi^{(2)}(n)=3 \varphi(n) .
$$

Le théorème suivant nous dit essentiellement que tout point extrémal de $\mathcal{O}_{X}$ se spécialise en une meilleure approximation de $\mathcal{O}_{n}$.

THÉORÈME 1. Soit $\varphi=U_{0}+U_{1} \Delta+U_{2} \Delta^{2}$ un point extrémal de $\mathcal{O}_{X}$, vérifiant $|\varphi|_{1} \leqslant 1$. On suppose qu'il existe un ensemble infini $E$ d'entiers $n$ tel que $U_{0}(n), U_{1}(n), U_{2}(n)$ soient entiers premiers dans leur ensemble. Alors, pour tout $n$ assez grand dans $E, \varphi(n)$ est un point extrémal de $\mathcal{O}_{n}$.

Preuve. Soient $(q, t)$ le couple d'entiers naturels définis par $|\varphi|_{2}=e^{2 q}$, $|\varphi|_{1}=e^{-t}$. En spécialisant on déduit

$$
\begin{aligned}
& |\varphi(n)|_{1} \leqslant c_{4} n^{-t}, \\
& |\varphi(n)|_{2} \leqslant c_{5} n^{2 q}
\end{aligned}
$$

où $c_{4}, c_{5}$ sont deux nombres réels indépendants de $n$ dans $E$.

On veut montrer que pour $n$ assez grand dans $E, \varphi(n)$ est une meilleure approximation de $\mathcal{O}_{n}$.

Soient $v_{0}, v_{1}, v_{2}$ des entiers non tous nuls tels que, en notant $\Delta=\Delta(n)$, $\psi_{n}=v_{0}+v_{1} \Delta+v_{2} \Delta^{2}$, on ait

$$
\left|\psi_{n}\right|_{i} \leqslant|\varphi(n)|_{i}, \quad i=1,2 .
$$

Nous allons montrer que s'il existe une infinité de $n$ dans $E$ et de $\psi_{n}$ vérifiant (5) alors on peut construire une fonction algébrique $\psi(X)$ telle que pour tout $n$ assez grand dans $E, \psi_{n}=\psi(n)$, et vérifiant

$$
|\psi(X)|_{i} \leqslant|\varphi(X)|_{i}, \quad i=1,2 .
$$

De l'hypothèse, $\varphi$ point extrémal et $\left(U_{0}(n), U_{1}(n), U_{2}(n)\right)$ triplet d'entiers primitifs, résultera alors $\psi_{n}= \pm \varphi(n)$ et le théorème 1 .
Notons $\psi_{n}^{\prime}=v_{0}+v_{1} \zeta \Delta+v_{2} \zeta^{2} \Delta^{2}$ et $\psi_{n}^{\prime \prime}=v_{0}+v_{1} \zeta^{2} \Delta+v_{2} \zeta \Delta^{2}\left(\zeta=e^{2 i \pi / 3}\right)$ les conjugués de $\psi$. De $3 v_{0}=\psi_{n}+\psi_{n}^{\prime}+\psi_{n}^{\prime \prime}$ on déduit la majoration

$$
\left|v_{0}\right| \leqslant \frac{1}{3}\left|\psi_{n}\right|+\frac{2}{3}\left|\psi_{n}^{\prime}\right| \leqslant\left(c_{4} / 3\right) n^{-t}+(2 / 3) \sqrt{c_{5}} n^{q} \leqslant c_{0} n^{q} .
$$

De même, à partir de $3 v_{1} \Delta=\psi_{n}+\zeta^{2} \psi_{n}^{\prime}+\zeta \psi_{n}^{\prime \prime}$ et de $3 v_{2} \Delta^{2}=\psi_{n}+\zeta \psi_{n}^{\prime}+\zeta^{2} \psi_{n}^{\prime \prime}$ on déduit

$$
\left|v_{j}\right| \leqslant c_{j} n^{q-j d}, \quad j=0,1,2,
$$

où $c_{0}, c_{1}, c_{2}$ sont des réels indépendants de $n$.

Nous pouvons alors écrire les entiers $v_{j}$ en base $n$, sous la forme

(8) $v_{j}=\sum_{i=0}^{q-j d} v_{i}^{(j)} n^{i} \quad$ avec $\quad-n / 2<v_{i}^{(j)} \leqslant n / 2 ; \quad i=0, \ldots, q-j d ; j=0,1,2$.

Posons maintenant

$$
Z_{j}=\sum_{i=0}^{q-j d} z_{i}^{(j)} X^{i}, \quad Z^{(q)}=\left(z_{q}^{(0)}, \ldots, z_{0}^{(0)}, z_{q-d}^{(1)}, \ldots, z_{0}^{(1)}, z_{q-2 d}^{(2)}, \ldots, z_{0}^{(2)}\right)
$$

où les $z_{i}^{(j)}$ sont des indéterminées.

Développons $\psi\left(X, Z^{(q)}\right)=Z_{0}+Z_{1} \Delta+Z_{2} \Delta^{2}$ en série de Laurent en $1 / X$ et notons $l_{k}^{(q)}$ le coefficient de $X^{k}$. $l_{k}^{(q)}$ est une forme linéaire en $Z^{(q)}$ dont les coefficients sont ceux de la ligne $k$ de la matrice $A_{q, \infty}$. On a

et

$$
A_{q, \infty} Z^{(q)}={ }^{t}\left(l_{q}^{(q)}\left(Z^{(q)}\right), l_{q-1}^{(q)}\left(Z^{(q)}\right), \ldots\right)
$$

$$
\begin{gathered}
l_{k}^{(q)}=0 \quad \text { pour } k>q, \\
l_{k}^{(q)}\left(Z^{(q)}\right)=\sum_{i=0}^{q} a_{k-i}^{(0)} z_{i}^{(0)}+\sum_{i=0}^{q-d} a_{k-i}^{(1)} z_{i}^{(1)}+\sum_{i=0}^{q-2 d} a_{k-i}^{(2)} z_{i}^{(2)} \quad \text { pour } k \leqslant q
\end{gathered}
$$

où on a posé

$$
\begin{gathered}
a_{0}^{(0)}=1, \quad a_{i}^{(0)}=0 \quad \text { si } i \neq 0 ; \quad a_{d}^{(1)}=1, \quad a_{i}^{(1)}=0 \quad \text { si } i>d ; \\
a_{2 d}^{(2)}=1, \quad a_{i}^{(2)}=0 \quad \text { si } i>2 d .
\end{gathered}
$$

En notant

$$
V^{(q)}=\left(v_{q}^{(0)}, \ldots, v_{0}^{(0)}, v_{q-d}^{(1)}, \ldots, v_{0}^{(1)}, v_{q-2 d}^{(2)}, \ldots, v_{0}^{(2)}\right)
$$

on a $\psi\left(n, V^{(q)}\right)=\psi_{n}$, donc

$$
\psi_{n}=\sum_{h \leqslant k \leqslant q} l_{k}^{(q)}\left(V^{(q)}\right) n^{k}+R_{h} n^{h} \quad \text { pour } h \leqslant q
$$

avec $R_{h}$ réel borné indépendamment de $n$.

D'autre part, il existe un entier $D$ indépendant de $n$ (dépendant de $q$ ) tel que $D l_{k}^{(q)}\left(V^{(q)}\right)$ soit entier pour $k=q, \ldots, 0,-1, \ldots,-t-1$. 
D'après (8) et (9) l'écriture en base $n$ de $D l_{k}^{(q)}\left(V^{(q)}\right)$ donne pour $n$ assez grand

$$
D l_{k}^{(q)}\left(V^{(q)}\right)=\lambda_{k} n+\mu_{k} \quad \text { avec }-n / 2<\mu_{k} \leqslant n / 2
$$

et avec $\lambda_{k}$ entier borné indépendamment de $n$ pour $q \geqslant k \geqslant-t-1$.

Regardons les conditions satisfaites par les $\lambda_{k}$ et les $\mu_{k}$ pour que $\psi_{n}$ vérifie (5) sachant (3).

D'après (10) avec $h=-t-1$ et (11) avec $k=-t-1$ on a

(12) $D \psi_{n}=\sum_{-t \leqslant k \leqslant q} D l_{k}^{(q)}\left(V^{(q)}\right) n^{k}+\left(\lambda_{-t-1} n+\mu_{-t-1}\right) n^{-t-1}+R_{-t-1}^{\prime} n^{-t-1}$

avec $R_{-t-1}^{\prime}$ borné indépendamment de $n$. (12) s'écrit encore

$$
n^{t} D \psi_{n}=\sum_{-t \leqslant k \leqslant q} D l_{k}^{(q)}\left(V^{(q)}\right) n^{t+k}+\lambda_{-t-1}+\left(\mu_{-t-1}+R_{-t-1}^{\prime}\right) n^{-1}
$$

D’autre part, (5) et (3) donnent $\left|n^{t} \psi_{n}\right| \leqslant c_{4}$ et donc d'après (13) et (11) cela permet d'écrire

(14) $\left|\sum_{-t \leqslant k \leqslant q}\left(\lambda_{k} n+\mu_{k}\right) n^{t+k}+\lambda_{-t-1}\right|=\left|\sum_{-t \leqslant k \leqslant q+1}\left(\mu_{k}+\lambda_{k-1}\right) n^{t+k}\right| \leqslant c_{4} D+1$

où l'on a posé $\mu_{q+1}=0$. On a alors $\left|\mu_{-t}+\lambda_{-t-1}\right| \leqslant c_{4} D+1, \mu_{-t+1}+\lambda_{-t}=0$ et de proche en proche jusqu'à $\mu_{q}+\lambda_{q-1}=0$ et $\lambda_{q}=0$. Ceci permet de réécrire (11) sous la forme

$$
\begin{aligned}
& D l_{k}^{(q)}\left(V^{(q)}\right)=\lambda_{k} n-\lambda_{k-1} \quad \text { pour } k=q, \ldots,-t+1, \\
& D l_{-t}^{(q)}\left(V^{(q)}\right)=\lambda_{-t} n-\lambda_{-t-1}+\delta
\end{aligned}
$$

avec $\delta$ borné indépendamment de $n$ par $c_{4} D+1$.

$$
\text { En terme matriciel on obtient en écrivant } \Lambda^{\prime}={ }^{\prime}\left(\lambda_{q}, \ldots, \lambda_{-t}\right) \text {, }
$$
$\Lambda^{\prime \prime}={ }^{t}\left(\lambda_{q-1}, \ldots, \lambda_{-t}, \lambda_{-t-1}-\delta\right)$

$$
D A_{q, q+t+1} V^{(q)}=n \Lambda^{\prime}-\Lambda^{\prime \prime} .
$$

Si $\delta$ et $\lambda_{k}$ pour $k=q, \ldots,-t-1$ sont fixés, $V^{(q)}$, d'ordre $3 q-3 d+3$, apparaît comme la solution du système linéaire (15), de rang $3 q-3 d+3$ d'après la proposition 4 . Nous en déduisons que les $v_{i}^{(j)}$ sont de la forme

$$
v_{i}^{(j)}=n v_{i}^{(j)}+v_{i}^{\prime \prime(j)}, \quad i=0, \ldots, q-j d ; j=0,1,2,
$$

où les $v_{i}^{(j)}$ et $v_{i}^{\prime \prime(j)}$ sont des rationnels indépendants de $n$. Leur dénominateur est donc borné indépendamment de $n$.

Posons

$$
\begin{gathered}
W_{j}(X)=\sum_{i=0}^{q-j d}\left(v_{i}^{(j)} X+v_{i}^{(j)}\right) X^{i}=\sum_{i=0}^{q-j d+1} w_{i}^{(j)} X^{i}, \quad j=0,1,2, \\
\psi(X)=W_{0}+W_{1} \Delta+W_{2} \Delta^{2}, \\
W^{(q+1)}=\left(w_{q+1}^{(0)}, \ldots, w_{0}^{(0)}, w_{q-d+1}^{(1)}, \ldots, w_{0}^{(1)}, w_{q-2 d+1}^{(2)}, \ldots, w_{0}^{(2)}\right) .
\end{gathered}
$$

On a alors pour $j=0,1,2$

$$
\begin{gathered}
w_{i}^{(j)}=w_{i-1}^{\prime(j)}+w_{i}^{\prime \prime(j)}, \quad i=0, \ldots, q-j d, \quad \text { et } \quad w_{q-j d+1}^{(j)}=v_{i}^{(j)}, \\
W_{j}(n)=v_{j} \quad \text { et } \quad \psi(n)=\psi_{n} .
\end{gathered}
$$

Si $\left(w_{q+1}^{(0)}, w_{q-d+1}^{(1)}, w_{q-2 d+1}^{(2)}\right) \neq 0$ on aurait $|\psi(X)|_{2}=e^{2(q+1)}$, ce qui contredit $|\psi(n)|_{2}=|\varphi(n)|_{2} \leqslant C_{5} n^{2 q}$ résultant de (4) et (5). Finalement, $W^{(q+1)}$ est essentiellement

$$
\tilde{W}^{(q)}=\left(w_{q}^{(0)}, \ldots, w_{0}^{(0)}, w_{q-d}^{(1)}, \ldots, w_{0}^{(1)}, w_{q-2 d}^{(2)}, \ldots, w_{0}^{(2)}\right) .
$$

On a donc $|\psi(X)|_{2} \leqslant e^{2 q}$, soit $|\psi(X)|_{2} \leqslant|\varphi(X)|_{2}$.

D'autre part, d'après (15), (16) et (17) on a $A_{q, q+t} \tilde{W}^{(q)}=0$, ce qui montre que $|\psi(X)|_{1} \leqslant e^{-t}$ et que $|\psi(X)|_{1} \leqslant|\varphi(X)|_{1}$.

Comme $\varphi$ est un point extrémal de $\mathcal{O}_{X}$, il existe $\lambda \in Z^{*}, \mu \in N^{*}$ premiers entre eux tels que $\mu \psi=\lambda \varphi$. Alors pour $j=0,1,2$ et $n$ assez grand dans $E$ on a $\mu W_{j}(n)=\mu v_{j}=\lambda U_{j}(n)$. Mais comme $U_{0}(n), U_{1}(n), U_{2}(n)$ sont premiers entre eux, on a $\mu= \pm 1$, et d'après (5), $\left|\psi_{n}\right|_{1}=|\lambda \varphi(n)|_{1} \leqslant|\varphi(n)|_{1}$, soit $\lambda= \pm 1$, ce qui termine la preuve du théorème 1 .

On montre maintenant que toute meilleure approximation de $\mathcal{O}_{n}$ $=\boldsymbol{Z}[\sqrt[3]{P(n)}]$, comprise entre deux points extrémaux voisins spécialisés, provient par spécialisation d'une face.

THÉORÈME 2. Avec les notations du théorème 1 soient $\varphi$ et $\pi$ deux points extrémaux voisins, $|\varphi|_{1}$ et $|\pi|_{1} \leqslant 1$ de $\mathcal{O}_{X}=Q[X, \Delta]$, tels que pour $n$ dans un ensemble infini $E, \varphi(n)$ et $\pi(n)$ soient des meilleures approximations de $\mathcal{O}_{n}$. Alors il existe une partition de $E$ en un nombre fini s de classes $E_{i}$, telles que pour tout $i$, $1 \leqslant i \leqslant s$, il existe un nombre fini $r_{i}$ de fonctions algébriques $\psi_{i}^{(j)}\left(1 \leqslant j \leqslant r_{i}\right)$ qui sont des faces de $\mathcal{O}_{X}$ et qui en se spécialisant donnent pour tout $n$ assez grand dans $E_{i}$ la chaine des meilleures approximations voisines joignant $\varphi(n) \dot{a} \pi(n)$.

Preuve. Soient $(q, t),\left(q^{\prime}, t^{\prime}\right)$ définis par $|\varphi|_{1}=e^{-t},|\pi|_{1}=e^{-t^{\prime}},|\varphi|_{2}=e^{2 q}$, $|\pi|_{2}=e^{2 q^{\prime}}$. On suppose $0<q<q^{\prime}$ et donc on a $t<t^{\prime}$.

\section{Posons}

$\varphi(X)=U_{0}(X)+U_{1}(X) \Delta+U_{2}(X) \Delta^{2} \quad$ et $\quad \pi(X)=T_{0}(X)+T_{1}(X) \Delta+T_{2}(X) \Delta^{2}$. En spécialisant on déduit

$$
\begin{aligned}
& |\varphi(n)|_{1} \leqslant c_{3} n^{-t}, \\
& |\varphi(n)|_{2} \sim c_{4} n^{2 q}, \\
& |\pi(n)|_{1} \leqslant c_{5} n^{-t^{\prime}}, \\
& |\pi(n)|_{2} \leqslant c_{6} n^{2 q^{\prime}},
\end{aligned}
$$

avec des constantes $c_{3}, c_{4}, c_{5}, c_{6}$ indépendantes de $n$. 
Par hypothèse $\varphi(n)$ et $\pi(n)$ sont deux meilleures approximations.

Si elles ne sont pas voisines considérons une meilleure approximation $\psi_{n}$ strictement intermédiaire et posons $\psi_{n}=v_{0}+v_{1} \Delta+v_{2} \Delta^{2}$. On a donc

$$
\begin{aligned}
& |\pi(n)|_{1}<|\psi(n)|_{1}<|\varphi(n)|_{1}, \\
& |\varphi(n)|_{2}<|\psi(n)|_{2}<|\pi(n)|_{2} .
\end{aligned}
$$

Puisque les conjugués de $\psi_{n}$ s'écrivent

$$
\psi_{n}^{\prime}=v_{0}+v_{1} \zeta \Delta+v_{2} \zeta^{2} \Delta^{2}, \quad \psi_{n}^{\prime \prime}=v_{0}+v_{1} \zeta^{2} \Delta+v_{2} \zeta \Delta^{2}
$$

on a

$$
3 v_{0}=\psi_{n}+\psi_{n}^{\prime}+\psi_{n}^{\prime \prime} .
$$

On obtient alors une majoration de $v_{0}$. De même pour $v_{1}$ et $v_{2}$. On a

$$
\left|v_{j}\right| \leqslant c_{j} n^{q^{\prime}-j d}, \quad j=0,1,2,
$$

avec $c_{0}, c_{1}, c_{2}$ independants de $n$.

On écrit alors $v_{0}, v_{1}, v_{2}$ en base $n$ sous la forme

(26) $v_{j}=\sum_{i=0}^{q^{\prime}-j d} v_{i}^{(j)} n^{i}, \quad-n / 2<v_{i}^{(j)} \leqslant n / 2, \quad i=0, \ldots, q^{\prime}-j d ; j=0,1,2$,

et (25) montre que $v_{q}^{(j)}-j d$ est majoré indépendamment de $n$.

En utilisant les mêmes notations et les mêmes arguments qu'au théorème 1, on obtient l'existence d'un entier $D$ tel que $D l_{k}^{\left(q^{\prime}\right)}\left(V^{q^{\prime}}\right)$ est entier pour $q^{\prime} \geqslant k \geqslant-t-1$

En écrivant ces entiers en base $n$, on a pour $n$ assez grand,

$$
D l_{k}^{\left(q^{\prime}\right)}\left(V^{\left(q^{\prime}\right)}\right)=\lambda_{k} n+\mu_{k}, \quad q^{\prime} \geqslant k \geqslant-t-1,
$$

avec $-n / 2<\mu_{k} \leqslant n / 2$ et $\lambda_{k}$ borné indépendamment de $n$.

Pour que $\psi_{n}$ vérifie (23) il faut avoir

$$
D A_{q^{\prime}, q^{\prime}+t+1} V^{\left(q^{\prime}\right)}=\Lambda^{\prime} n-\Lambda^{\prime \prime}
$$

où $\Lambda^{\prime}={ }^{t}\left(\lambda_{q^{\prime}}, \ldots, \lambda_{-t}\right), \Lambda^{\prime \prime}={ }^{t}\left(\lambda_{q^{\prime}-1}, \ldots, \lambda_{-t}, \lambda_{-t-1}-\delta\right)$ avec $\lambda_{q^{\prime}}=0$ et $|\delta|$ $\leqslant D c_{3}+1$.

Montrons maintenant que si $\psi_{n}$ est une meilleure approximation entre $\varphi(n)$ et $\pi(n)$ alors $v_{q^{\prime}}^{(0)}, v_{q^{\prime}-d}^{(1)}, v_{q^{\prime}-2 d}^{(2)}$ sont bornés indépendamment de $n$.

L'égalité

$$
\left|\psi_{n}\right|_{2}=\frac{3}{2}\left(v_{0}^{2}+v_{1}^{2} \Delta^{2}+v_{2}^{2} \Delta^{4}\right)-\frac{1}{2} \psi_{n}^{2}
$$

qui est facile à vérifier, permet d'écrire

$$
\frac{3}{2} \sum_{j=0}^{2} v_{q^{\prime}}^{(j)^{2}}-j d=\frac{3}{2} \sum_{j=0}^{2}\left(v_{q^{\prime}-j d}^{\left(j j^{2}\right.}-\frac{v_{j}^{2} \Delta^{2 j}}{n^{2 q^{\prime}}}\right)+\frac{\left|\psi_{n}\right|_{2}}{n^{2 q^{\prime}}}+\frac{1}{2} \frac{\psi_{n}^{2}}{n^{2 q^{\prime}}},
$$

et en tenant compte de (26), (23), (24), (22), (25), 19 on obtient

$$
\frac{3}{2} \sum_{j=0}^{2} v_{q^{\prime}-j d}^{(j)^{2}} \leqslant 3 G\left(\left|v_{q^{\prime}}^{(0)}\right|+\left|v_{q^{\prime}-d}^{(1)}\right|+\left|v_{q^{\prime}-2 d}^{(2)}\right|+1\right)
$$

avec $G$ borné indépendamment de $n$.

Les entiers $v_{q^{\prime}-j d}^{(j)}$ sont donc bornés. $\Lambda^{\prime}$ et $\Lambda^{\prime \prime}$ sont aussi en nombre fini. Il existe donc un choix de $v_{q^{(j)}-j d}(j=0,1,2), \Lambda^{\prime}, \Lambda^{\prime \prime}$ et un ensemble infini $E_{1}$ tels que pour tout $n$ dans $E_{1}, \psi_{n}$ et $v_{0}, v_{1}, v_{2}$ correspondants vérifient (28). Mais puisque les $v_{q^{\prime}}^{(j)}-j d$ sont fixés, les inconnues dans $V^{(q+1)}$ se réduisent à $\tilde{V}^{\left(q^{\prime}-1\right)}=\left(v_{q^{\prime}-1}^{(0)}, \ldots ; v_{q^{\prime}-1-d}^{(1)}, \ldots ; v_{q^{\prime}-1-2 d}^{(2)}, \ldots v_{0}^{(2)}\right)$ et on a:

$$
D A_{q^{\prime}-1, q^{\prime}+t} \tilde{V}^{(q)}=\tilde{\Lambda}^{\prime} n-\tilde{\Lambda}^{\prime \prime}-D\left(v_{q^{\prime}-d}^{(1)} A^{(1)}+v_{q^{\prime}-2 d}^{(2)} A^{(2)}\right)
$$

$$
\begin{gathered}
\tilde{\Lambda}^{\prime}={ }^{t}\left(\lambda_{q^{\prime}-1}, \ldots, \lambda_{-t}\right), \quad \tilde{\Lambda}^{\prime \prime}={ }^{t}\left(\lambda_{q^{\prime}-2}, \ldots, \lambda_{-t-1}-\delta\right), \\
A^{(1)}={ }^{t}\left(a_{d-1}^{(1)} \ldots a_{d-q^{\prime}-t}^{(1)}\right), \quad A^{(2)}={ }^{t}\left(a_{2 d-1}^{(2)} \ldots a_{2 d-q^{\prime}-t}^{(2)}\right) .
\end{gathered}
$$

D'après la proposition 4(iii) le rang de ce système est égal au nombre d'inconnues $3\left(q^{\prime}-d+1\right)$. On peut donc écrire

(32) $\quad v_{i}^{(j)}=n v_{i}^{(j)}+v_{i}^{\prime \prime(j)}, \quad 0 \leqslant i \leqslant q^{\prime}-j d ; \quad v_{q}^{\prime(j)}-j d=0 ; \quad j=0,1,2$,

où les $v_{i}^{\prime(j)}$ et $v_{i}^{\prime \prime(j)}$ sont des rationnels indépendants de $n$ dans $E_{1}$. Leur dénominateur est donc borné.

Posons

$$
W_{j}(X)=\sum_{i=0}^{q^{\prime}-j d}\left(v_{i}^{\prime(j)} X+v_{i}^{\prime \prime(j)}\right) X^{i}:=\sum_{i=0}^{q^{\prime}-j d} w_{i}^{(j)} X^{i} \quad \text { et } \quad \psi=W_{0}+W_{1} \Delta+W_{2} \Delta^{2} .
$$

On a donc pour tout $n$ dans $E_{1}$

$$
\psi(n)=\psi_{n} .
$$

Les éléments du noyau de $A_{q^{\prime}-1, q^{\prime}+t}$ n'interviennent pas dans $\psi$ d'après la proposition 4. Il nous reste à montrer que $\psi(X)$ est une face de $\mathcal{O}_{X}$.

En tenant compte de (31), (32) et de la première équation de (28), $v_{q}^{(0)}+v_{q^{\prime}-d}^{(1)}+v_{q^{\prime}-2 d}^{(2)}=-\lambda_{q}$, le $3\left(q^{\prime}-d+1\right)$-uple $W^{\left(q^{\prime}\right)}=\left(w_{q^{\prime}}^{(0)} \ldots ; w_{q^{\prime}-d}^{(1)} \ldots\right.$ $\left.\ldots ; w_{q^{\prime}-2 d}^{(2)} \ldots w_{0}^{(2)}\right)$ vérifie

$$
A_{q^{\prime}, q^{\prime}+t} W^{\left(q^{\prime}\right)}=0,
$$

ce qui entraîne $|\psi(X)|_{1} \leqslant e^{-t}=|\varphi(X)|_{1}$.

Pour obtenir $|\psi(X)|_{2}$ on montre que $\left(w_{q^{\prime}}^{(0)}, w_{q^{\prime}-d}^{(1)}, w_{q^{\prime}-2 d}^{(2)}\right) \neq 0$.

Dans le cas contraire nous allons obtenir $\psi=\lambda \varphi$, ce qui sera contradictoire avec (23) et (24).

Le $3\left(q^{\prime}-d\right)$-uple $\tilde{W}^{\left(q^{\prime}-1\right)}=\left(w_{q^{\prime}-1}^{(0)} \ldots ; w_{q^{\prime}-1-d}^{(1)} \ldots ; w_{q^{\prime}-1-2 d}^{(2)} \ldots w_{0}^{(2)}\right)$ vérifie, d'après (34), $A_{q^{\prime}-1, q^{\prime}+t-1} \tilde{W}^{\left(q^{\prime}-1\right)}=0$. On a donc $|\psi(X)|_{1} \leqslant|\varphi(X)|_{1}$. D'autre part, $|\psi(X)|_{2} \leqslant|\varphi(X)|_{2}$. Mais puisque $\varphi \in \mathscr{E}_{X}$ et que $0 \neq \psi \in \mathcal{O}_{X}$, il existe 
$\lambda \in Q^{*}$ tel que $\psi=\lambda \varphi$. Par spécialisation on obtient $\psi(n)=\psi_{n}=\lambda \varphi(n)$, ce qui contredit (23) ou (24). Ceci prouve que $|\psi(X)|_{2}=e^{2 q^{\prime}}=|\pi|_{2}$.

Nous sommes maintenant en mesure de montrer que $\psi$ est une face de $\mathcal{O}_{X}$. Soit $\xi \in \mathcal{O}_{X} \backslash 0$ tel que

$$
|\xi|_{i} \leqslant|\psi|_{i}, \quad i=1,2 .
$$

Puisque $\varphi$ et $\pi$ sont des points extrémaux voisins et que $|\xi|_{i} \leqslant \operatorname{Max}\left(|\varphi|_{i},|\pi|_{i}\right)$ on a $|\xi|_{1}=|\varphi|_{1}$ et donc $|\xi|_{1}=|\psi|_{1}$ ou $|\xi|_{2}=|\pi|_{2}$ et donc $|\xi|_{2}=|\psi|_{2} \cdot \psi$ est donc une face, ce qui termine la preuve du théorème 2 .

IV. Application: Etude du calibre de l'anneau $\mathcal{O}_{n}$. On sait que pour tout $n \in \tilde{Z}$, on peut définir l'ensemble quotient $\mathscr{E}_{n} / \mathscr{U}_{n}$ et que pour $n$ fixé cet ensemble est fini; on appelle son cardinal le calibre de $\mathcal{O}_{n}$ (voir II.a et [4]).

D'autre part, on peut, de la même façon, définir l'ensemble quotient $\mathscr{E}_{X} /\left(\mathscr{U}_{X} / Q^{*}\right)$ où $\mathscr{U}_{X} / Q^{*}$ est le groupe des unités modulo $Q^{*}$.

On le note, par abus de notation, $\mathscr{E}_{X} / \mathscr{U}_{X}$ (voir II.b) et on appelle calibre de $\mathcal{O}_{X}$ son cardinal.

On va déduire des théorèmes 1 et 2 et d'une hypothèse sur le calibre de $\mathcal{O}_{X}$ des renseignements sur le calibre de $\mathcal{O}_{n}$ lorsque nous faisons varier l'entier $n$.

Pour $n \in \tilde{Z}$, on note par $K_{n}$ le corps de nombres $Q(\sqrt[3]{P(n)})$.

THÉORÈME 3. Si le graphe quotient $\mathscr{E}_{X} / \mathscr{U}_{X}$ est infini alors

$$
\lim _{n \in \mathcal{Z}, n \rightarrow \infty}\left|\mathscr{E}_{n}\right| \mathscr{U}_{n} \mid=+\infty .
$$

Preuve. Soit $k$ un entier positif. D'après le théorème 2 , il existe une partition $E_{1}, \ldots, E_{s_{k}}$ de $\tilde{Z}$ et des fonctions algébriques $\varphi_{i}^{(j)}\left(1 \leqslant j \leqslant s_{k}\right.$, $0 \leqslant i \leqslant k)$ du corps $K$ telles que pour tout $i, j$ et $n$ assez grand dans $E_{j}$, la chaine des $k+1$ premières meilleures approximations de $\mathcal{O}_{n}$ de première valeur absolue inférieur à 1 soit

$$
1=\varphi_{\gamma}^{(j)}(n), \ldots, \varphi_{k}^{(j)}(n) .
$$

Puisque $\left|\mathscr{E}_{X} / \mathscr{U}_{X}\right|=\infty$ la proposition 2 montre que les $\varphi_{i}^{(j)}(X)$ ne sont pas des unités. Alors pour tout $i \in[0, k], j \in\left[1, s_{k}\right], N_{K_{X} / Q(X)} \varphi_{i}^{(j)}(X)$ n'est pas une constante rationnelle et donc pour $n$ assez grand dans $E_{j}, N_{K_{n} / \mathbf{Q}} \varphi_{i}^{(j)}(n) \neq \pm 1$ et $\varphi_{i}^{(j)}(n)$ n'est pas une unité de $\mathcal{O}_{n}$. Alors $\left|\mathscr{E}_{n}\right| \mathscr{U}_{n} \mid \geqslant k$ et le théorème 3 est démontré.

THÉORÈME 4. Si le graphe quotient $\mathscr{E}_{X} / \mathscr{U}_{X}$ est fini, considérons $\varepsilon(X)$ l'unité fondamentale de $\mathcal{O}_{X}$ de norme 1, E lensemble des entiers $n$ de $\tilde{Z}$ tels que $\varepsilon(n)$ soit un entier de $K_{n}$. Alors on a

$$
\begin{aligned}
& \lim _{n \rightarrow \infty, n \in \mathbb{Z} \backslash E}\left|\mathscr{E}_{n}\right| \mathscr{U}_{n} \mid=\infty . \quad(\text { si } \tilde{Z} \backslash E \text { est infini), } \\
& \left|\mathscr{E}_{X}\right| \mathscr{U}_{X}^{(1)}\left|\leqslant \varliminf_{n \in E}\right| \mathscr{E}_{n} / \mathscr{U}_{n}\left|\leqslant \varlimsup_{n \in E}\right| \mathscr{E}_{n} / \mathscr{U}_{n} \mid<+\infty .
\end{aligned}
$$

Preuve.

1. Montrons (36). On suppose que l'ensemble $\tilde{Z} \backslash E$, que l'on note $E^{c}$, est infini. Soit $k$ un entier positif. D’après le théorème 2 , il existe une partition $E_{1}, \ldots, E_{s_{k}}$ de $\tilde{Z}$ et des fonctions $\varphi_{i}^{(j)}(X)$ pour $1 \leqslant j \leqslant s_{k}$ et $0 \leqslant i \leqslant k$, telles que pour tout $n$ assez grand dans $E_{j}$ les $k+1$ premières meilleures approximations de $\mathcal{O}_{n}$ de première valeur absolue inférieure à 1 sont $1=\varphi_{0}^{(j)}(n), \varphi_{1}^{(j)}(n), \ldots, \varphi_{k}^{(j)}(n)$. Supposons que l'ensemble $E_{j} \cap E^{c}$ des entiers de $E_{j}$ n'appartenant pas à $E$ est infini. Pour tout $i$ dans $[1, k]$ et tout $n$ assez grand dans $E_{j} \cap E^{c}$, $N_{K_{n} / \mathbf{Q}} \varphi_{i}^{(j)}(n) \neq \pm 1$. En effet, raisonnons par l'absurde et supposons qu'il existe $i$ dans $[1, k]$, tel que pour une infinité de $n$ dans $E_{j} \cap E^{c}, N_{K_{n} / Q} \varphi_{i}^{(j)}(n)= \pm 1$. $\varphi_{i}^{(j)}(X)$ vérifie alors $N_{K / Q(X)} \varphi_{i}^{(j)}(X)= \pm 1$ et est donc une unité non triviale de $K_{X}$ de norme \pm 1 . Il existe alors un entier $l \geqslant 1$ tel que $\varphi_{i}^{(j)}= \pm \varepsilon^{l}$. En spécialisant on obtient

$$
\varphi_{i}^{(j)}(n)= \pm \varepsilon^{l}(n), \quad \forall n \in E_{j} \cap E^{c} .
$$

D'autre part, d'après le théorème $2, \varphi_{i}^{(j)}(n) \in \mathcal{O}_{n}$, pour tout $n$ dans $E_{j}$. Or $\mathcal{O}_{n} \subset K_{n}$ et $\varepsilon^{l}(n) \in B_{n}$, l'anneau des entiers de $K_{n}$. Mais ceci implique $\varepsilon(n) \in B_{n}$ et contredit l'hypothèse $n \notin E$.

On a donc montré que $N_{K_{n} / \mathbf{Q}} \varphi_{i}^{(j)}(n) \neq \pm 1$ sauf pour un nombre fini de $n$.

Ceci montre que, pour tout $n$ assez grand dans $E_{j} \cap E^{c}$, les $k+1$ premières meilleures approximations de $\mathcal{O}_{n}, \varphi_{0}^{(j)}(n), \ldots, \varphi_{k}^{(j)}(n)$, ne sont pas des unités et donc que $\left|\mathscr{E}_{n}\right| \mathscr{U}_{n} \mid \geqslant k$, ce qui prouve la première partie du théorème.

2. Montrons maintenant l'inégalité droite de (37). Supposons $E$ de cardinal infini et posons

$$
\varepsilon(X)=U_{0}(X)+U_{1}(X) \Delta+U_{2}(X) \Delta^{2}, \quad U_{i}(X) \in Q[X], \quad i=0,1,2 .
$$

On peut écrire $\varepsilon(X)$ sous la forme

$$
\varepsilon(X)=U_{0}(X)+\frac{W_{1}(X)}{m} \Delta+\frac{W_{2}(X)}{m} \Delta^{2}
$$

où $m$ est un entier $\geqslant 1$ et où $W_{1}(X), W_{2}(X)$ sont deux polynômes à valeurs entières sur $\boldsymbol{Z}$. Soit $n \in \boldsymbol{Z}$. On peut écrire

$$
\varepsilon(n)=U_{0}(n)+\frac{V_{1}}{m_{n}} \Delta_{n}+\frac{V_{2}}{m_{n}} \Delta_{n}^{2}
$$

où $m_{n}, V_{1}, V_{2}$ sont trois entiers premiers entre eux dans leur ensemble. $m_{n}$ divise $m$ et est donc borné indépendamment de $n$.

Nous allons poursuivre la démonstration sous forme de lemmes.

LEMME 1. Si $\varepsilon(n)$ est un entier algébrique alors $3 U_{0}(n)$ est un entier relatif et $m_{n}^{3}$ divise $27 P(n)$.

Preuve. 1. $\operatorname{Tr}_{K_{n} / \boldsymbol{Q}}(\varepsilon(n))=3 U_{0}(n) \in \boldsymbol{Z}$. 
2. On calcule la somme

$$
\varepsilon(n)+\zeta^{2} \sigma(\varepsilon(n))+\zeta \sigma\left(\varepsilon^{2}(n)\right)=3 V_{1} \Delta / m_{n}
$$

où $\sigma$ est l'isomorphisme de $K_{n}$ dans $C$ défini par $\sigma(\Delta)=\zeta \Delta$.

Puis sa norme:

$$
N_{K_{n} / \mathbf{Q}}\left(\frac{3 V_{1} \Delta}{m_{n}}\right)=\frac{27 V_{1}^{3}}{m_{n}^{3}} P(n) \in \boldsymbol{Z}
$$

De même,

$$
N_{K_{n} / \mathbf{Q}}\left(\frac{3 V_{2} \Delta}{m_{n}}\right)=\frac{27 V_{2}^{3}}{m_{n}^{3}} P(n) \in \boldsymbol{Z},
$$

et comme les trois entiers $V_{1}, V_{2}, m_{n}$ sont premiers entre eux, $m_{n}^{3}$ divise $27 P(n)$.

\section{Lemme 2. Soit $n \in E$.}

1. Si $m_{n}^{3}$ divise $P(n)$ et si on pose $\Delta_{n}^{*}=\sqrt[3]{P(n) / m_{n}^{3}}=\Delta_{n} / m_{n}$, alors $\varepsilon(n) \in \boldsymbol{Z}\left[\Delta_{n}^{*}\right]$.

2. Si $m_{n}^{3}$ ne divise pas $P(n)$ (i.e. 3 divise $m_{n}$ et $\left(m_{n} / 3\right)^{3}$ divise $P(n)$ ). Posons

$$
\Delta_{n}^{*}=\sqrt[3]{\frac{27 P(n)}{m_{n}^{3}}}=\frac{3 \Delta_{n}}{m_{n}} \quad \text { et } \quad \frac{27 P(n)}{m_{n}^{3}}=f g^{2} k^{3}
$$

où $f$ est un entier sans facteur carré, $g$ est un entier sans facteur carré, $f$ et $g$ sont premiers entre eux et $k$ est un entier.

Alors on $a$ :

(i) Si 3 divise g alors $\varepsilon^{3}(n) \in \mathbf{Z}\left[\Delta_{n}^{*}\right]$.

(ii) Si 3 ne divise pas $g$ alors $\varepsilon^{2}(n) \in \boldsymbol{Z}\left[\Delta_{n}^{*}\right]$.

Preuve. Rappelons la caractérisation d'un entier dans un corps cubique pur:

Soit $\delta=\sqrt[3]{f g^{2}}$ où $f$ est sans facteur carré, $g$ est sans cube et les entiers $f$ et $g$ sont premiers entre eux. Posons $\bar{\delta}=\sqrt[3]{f^{2} g}$. Alors on a:

(a) $\operatorname{Si} f g^{2} \not \equiv \pm 1(\bmod 9)$ alors $(1, \delta, \delta)$ est une $Z$-base d'entiers.

(b) $\mathrm{Si} f g^{2} \equiv \pm 1(\bmod 9)$ alors $(1, \delta, \varrho=(1+f \delta+g \bar{\delta}) / 3)$ est une $Z$-base d'entiers.

Enfin, rappelons le résultat suivant:

(c) $\mathrm{Si}(x+y \delta+z \delta) / 3$ est un entier $(x, y, z \in \boldsymbol{Z})$ et si 3 divise $x$ ou $y$ ou $z$ alors 3 divise $x$ et $y$ et $z$.

Montrons maintenant le lemme 2.
1. Si $m_{n}^{3}$ divise $P(n)$, posons $P(n) / m_{n}^{3}=f g^{2} k^{3}$ où $f, g$ et $k$ sont des entiers définis comme précédemment. On a

$$
\varepsilon(n)=3 U_{0} / 3+V_{1} \Delta_{n}^{*}+m_{n} V_{2} \Delta_{n}^{*^{2}}=3 U_{0} / 3+V_{1} k \delta+m_{n} V_{2} k^{2} g \delta
$$

et d'après (c) on en déduit que $U_{0}$ est entier et donc $\varepsilon(n) \in \boldsymbol{Z}\left[\Delta_{n}^{*}\right]$.

2. Si $\left(m_{n} / 3\right)^{3}$ divise $P(n)$ et $m_{n}^{3}$ ne divise pas $P(n)$ on a

$$
\begin{aligned}
& \varepsilon(n)=U_{0}+\frac{V_{1}}{3} \Delta_{n}^{*}+\frac{V_{2}}{3} \frac{m_{n}}{3} \Delta_{n}^{*^{2}}, \\
& \varepsilon(n)=\frac{3 U_{0}}{3}+\frac{V_{1} k}{3} \delta+\frac{V_{2}}{3} \frac{m_{n}}{3} k^{2} g \delta .
\end{aligned}
$$

(i) Si 3 divise $g$, alors $V_{2} m_{n} k^{2} g / 3^{2}$ est un entier et d'après (c), $U_{0}$ est entier et 3 divise $V_{1}$. Calculons $\varepsilon^{3}$ :

$$
\begin{aligned}
\varepsilon^{3}(n)= & U_{0}^{3}+\left(\frac{V_{1}}{3}\right)^{3} \Delta_{n}^{*^{3}}+\frac{V_{2}^{3}}{3^{3}}\left(\frac{m_{n}}{3}\right)^{3} f^{2} g^{4} k^{6}+3 U_{0} \frac{V_{1}}{3} \frac{V_{2}}{3} \frac{m_{n}}{3} \Delta_{n}^{*^{3}} \\
& +3\left(U_{0}^{2} \frac{V_{1}}{3}+\left(\frac{V_{1}^{2}}{3}\right) \frac{V_{2}}{3} \frac{m_{n}}{3} \Delta_{n}^{*^{3}}+U_{0}\left(\frac{V_{2}^{2}}{3^{2}}\right)\left(\frac{m_{n}}{3}\right)^{2} f g^{2} k^{3}\right) \Delta_{n}^{*} \\
& +3\left(U_{0}^{2} \frac{V_{2}}{3}+\frac{m_{n}}{3}+\frac{V_{1}}{3} \frac{V_{2}^{2}}{3^{2}}\left(\frac{m_{n}}{3}\right)^{2} f g^{2} k^{3}+U_{0}\left(\frac{V_{1}}{3}\right)^{2}\right) \Delta_{n}^{*^{2}}
\end{aligned}
$$

et comme 3 ne divise $g$, on voit que $\varepsilon^{3}(n) \in \boldsymbol{Z}\left[\Delta_{n}^{*}\right]$.

(ii) Supposons que 3 ne divise pas $g$. D'après (39) et (c), si 3 divise $3 U_{0}$ ou $V_{1}$ ou $V_{2}$ le résultat est trivial car $\varepsilon(n) \in Z\left[\Delta_{n}^{*}\right]$ et donc aussi $\varepsilon^{2}(n)$.

Si 3 ne divise pas $3 U_{0}, V_{1} k, V_{2}\left(m_{n} / 3\right) k^{2} g$ et que $\varepsilon(n)$ est entier alors d'après (a) et (b) nous sommes dans un corps cubique pur dont une base d'entiers est $(1, \delta, \varrho)$ (on a donc $f g^{2} \equiv \pm 1(\bmod 9)$ ). Ceci permet d'écrire

$$
\varepsilon(n)=x+y \delta+z \varrho, \quad x, y, z \in \boldsymbol{Z} .
$$

Montrons que $\varepsilon^{2}(n) \in \boldsymbol{Z}+\boldsymbol{Z} \delta+\boldsymbol{Z} \delta$.

On peut écrire $\varepsilon^{2}(n)=u+v \delta+w \varrho$ avec, $u, v, w \in \boldsymbol{Z}$. Comme $f g^{2} \equiv \pm 1$ ( $\bmod 9)$ on sait que l'idéal (3) dans le corps de nombres $\boldsymbol{Q}\left(\zeta, \Delta_{n}\right)$ se décompose Sous la forme (3) $=P_{1}^{2} P_{2}^{2} P_{3}^{2}$, les $P_{i}$ étant des idéaux premiers distincts de l'anneau des entiers dont le corps résiduel est $\boldsymbol{Z} / 3 \boldsymbol{Z}$.

D'autre part, $\varepsilon(n) \notin P_{i}$ car $\varepsilon(n)$ est une unité. On en déduit les congruences

$$
\sigma^{j}\left(\varepsilon^{2}(n)\right) \equiv 1\left(\bmod P_{i}\right), \quad j=0,1,2,
$$

et en sommant ces trois congruences,

$$
3 u+w \equiv 0(\bmod 3) .
$$

Donc 3 divise $w$, i.e. $\varepsilon^{2}(n) \in \boldsymbol{Z}+\boldsymbol{Z} \delta+\boldsymbol{Z} \bar{\delta}$.

3- Acta Arithmetica 59.3 
Reste à voir que $\varepsilon^{2}(n) \in \boldsymbol{Z}\left[\Delta_{n}^{*}\right]$.

En effet, on a

$$
\begin{aligned}
\varepsilon^{2}(n)= & U_{0}^{2}+2 \frac{V_{1}}{3} \frac{V_{2}}{3} \frac{m_{n}}{3} f g^{2} k^{3}+\left(2 U_{0} \frac{V_{1}}{3}+\frac{V_{2}^{2}}{3^{2}}\left(\frac{m_{n}}{3}\right)^{2} \Delta_{n}^{*^{3}}\right) \Delta_{n}^{*} \\
& +\left(2 U_{0} \frac{V_{2}}{3} \frac{m_{n}}{3}+\frac{V_{1}^{2}}{3^{2}}\right) \Delta_{n}^{*^{2}}
\end{aligned}
$$

et donc

$$
\begin{aligned}
u+\frac{w}{3} & =U_{0}^{2}+2 \frac{V_{1}}{3} \frac{V_{2}}{3}\left(\frac{m_{n}}{3}\right) f g^{2} k^{3} \in \boldsymbol{Z}, \\
v+\frac{f w}{3} & =\left(2 U_{0} \frac{V_{1}}{3}+\frac{V_{2}^{2}}{3^{2}}\left(\frac{m_{n}}{3}\right)^{2} \Delta_{n}^{*^{3}}\right) k \in \boldsymbol{Z}, \\
\frac{g w}{3} & =\left(2 U_{0} \frac{V_{2}}{3} \frac{m_{n}}{3}+\frac{V_{1}^{2}}{3^{2}}\right) k^{2} g \in \boldsymbol{Z},
\end{aligned}
$$

et comme 3 ne divise pas $k$ et $g$ on en déduit que $\varepsilon^{2}(n) \in \boldsymbol{Z}\left[\Delta_{n}^{*}\right]$.

Lemme 3. Soit $n \in E$. Alors il existe un entier $k \geqslant 1$ borné indépendamment de $n$ tel que $\varepsilon^{k}(n) \in \boldsymbol{Z}\left[\Delta_{n}\right]$.

Preuve. Il suffit de montrer que dans un corps cubique pur $Q(\Delta)$, $\Delta^{3}=D \in \boldsymbol{Z}, D$ non cube ( $D$ n'est pas supposé sans cube), si on considère l'ordre $A_{l}=Z[l \Delta]$, où $l$ est entier $\geqslant 1$, lindice que nous noterons $v(l)$ du groupe des unités $G_{l}$ de $A_{l}$ par rapport à $G$ groupe des unités de $Z[\Delta]$ est borné par une constante indépendante de $D$.

En effet, nous savons que $\varepsilon^{6}(n) \in \boldsymbol{Z}\left[\Delta_{n}^{*}\right]$ et que $\Delta_{n}^{*}=\Delta_{n} / m_{n}$ ou $3 \Delta_{n} / m_{n}$ d'après le lemme 2. Donc si nous prenons $l=m_{n}$ on en déduira que $\varepsilon(n)^{6 v\left(m_{n}\right)} \in \boldsymbol{Z}\left[\Delta_{n}\right]$, avec $v\left(m_{n}\right)$ borné indépendamment de $\Delta_{n}^{*}$. Comme $m_{n}$ est borné indépendamment de $n\left(m_{n}\right.$ divise $\left.m\right)$ on en déduit le lemme 3 .

Nous allons donc faire une étude de cet indice $v(l)$.

On peut supposer que $l$ est une puissance d'un nombre premier car si $l_{1}$ et $l_{2}$ sont deux entiers premiers entre eux, on a l'égalité

$$
v\left(l_{1} l_{2}\right)=\text { p.p.c.m. }\left(v\left(l_{1}\right), v\left(l_{2}\right)\right) \text {. }
$$

Lemme 4. Soient $l$ un nombre premier et $\alpha$ un entier naturel non nul. (i) $\mathrm{Si} l$ ne divise pas le discriminant du corps alors $v\left(l^{\alpha}\right)$ divise $(l-1) l^{2 \alpha-1}$ ou $\left(l^{2}-1\right) l^{2 \alpha-1}$ ou $\left(l^{3}-1\right) l^{2 \alpha-1}$.

(ii) Si l divise le discriminant du corps alors $v\left(l^{\alpha}\right)$ divise $l^{2 \alpha}$

Preuve. (i) Traitons d'abord le cas $\alpha=1$. L'idéal (l) se décompose en au plus trois idéaux premiers et si on désigne par $\varepsilon$ une unité de $Z[\Delta]$, on a
(a) $\varepsilon^{l-1} \equiv 1(\bmod (l))$ si $(l)=\mathfrak{p}_{1} \mathfrak{p}_{2} \mathfrak{p}_{3}$,
(b) $\varepsilon^{l^{2}-1} \equiv 1(\bmod (l))$ si $(l)=\mathrm{p}_{1} \mathrm{p}_{2}$,
(c) $\varepsilon^{l^{3}-1} \equiv 1(\bmod (l))$ si $(l)=\mathfrak{p}_{1}$

On en déduit que l'indice $\left[G: G^{(t)}\right]$ divise respectivement $l-1, l^{2}-1, l^{3}-1$; on note par $G^{(l)}$ le groupe des unités de l'ordre $A^{(l)}=\boldsymbol{Z}+\boldsymbol{Z} l \Delta+\boldsymbol{Z} l \Delta^{2}$.

Posons $i=\left[G: G^{(l)}\right]$ et $\varepsilon^{i}=x+y l \Delta+z l \Delta^{2} \in A^{(l)}$. Puis calculons $\left(\varepsilon^{i}\right)^{l}$ :

$$
\varepsilon^{i l}=\sum_{i_{0}+i_{1}+i_{2}=l}\left(\begin{array}{c}
l \\
i_{0}, i_{1}, i_{2}
\end{array}\right) x^{i_{0}} y^{i_{1}} z^{i_{2}} l^{i_{1}+i_{2}} \Delta^{i_{1}+2 i_{2}} .
$$

Pour avoir le résultat pour $\alpha=1$, il faut montrer que $\varepsilon^{i l} \in A_{l}$. Posons

$$
t_{i_{0}, i_{1}, i_{2}}=\left(\begin{array}{c}
1 \\
i_{0}, i_{1}, i_{2}
\end{array}\right) x^{i_{0}} y^{i_{1}} z^{i_{2}} l^{i_{1}+i_{2}} D^{\left.\left[i_{1}+2 i_{2}\right) / 3\right]}
$$

où $[\gamma]$ désigne la partie entière de $\gamma$.

Si $i_{1}+2 i_{2} \geqslant 3$ alors $v_{l}\left(t_{i_{0}, i_{1}, i_{2}}\right) \geqslant 3, v_{l}$ désigne la valuation $l$-adique. $\subset A_{l}$.

Si $i_{1}+2 i_{2}<3$ alors $v_{l}\left(t_{l-1,1,0}\right) \geqslant 2$, et $v_{l}\left(t_{l-1,0,1}\right) \geqslant 2$. Par suite $\varepsilon^{i t} \in A^{\left(t^{2}\right)}$

Reste le cas $\alpha>1$. On part de l'égalité

$$
\left[G: G^{\left(l^{2 \alpha}\right)}\right]=\left[G: G^{(l)}\right]\left[G^{(l)}: G^{\left(l^{2 \alpha}\right)}\right] \ldots\left[G^{\left(2^{2 \alpha-1}\right)}: G^{(2 \alpha)}\right],
$$

on montre comme précédemment que l'indice $\left[G^{\left(l^{l i}\right)}: G^{\left(t^{i+1}\right)}\right]$ divise $l$ pour $1 \leqslant i \leqslant 2 \alpha-1$, et l'inclusion $G^{\left(l^{2 \alpha}\right)} \subset G_{l^{\alpha}}$ permet de conclure.

(ii) Si $l=3$, il suffit de remarquer que si $\varepsilon \in \boldsymbol{Z}[\Delta]$ alors $\varepsilon^{3} \in A^{(3)}$, $\varepsilon^{32} \in A^{(32)}, \ldots$

Si $l \neq 3$, soit $\varepsilon \in G$. On pose $\varepsilon=x+y \Delta+z \Delta^{2}$ et on calcule $\varepsilon^{l}$ :

$$
\varepsilon^{l}=\sum_{i_{0}+i_{1}+i_{2}=l}\left(\begin{array}{c}
l \\
i_{0}+i_{1}+i_{2}
\end{array}\right) x^{i_{0}} y^{i_{1}} z^{i_{2}} \Delta^{i_{1}+2 i_{2}}
$$

On pose aussi

$$
t_{i_{0}, i_{1}, i_{2}}=\left(\begin{array}{c}
1 \\
i_{0}, i_{1}, i_{2}
\end{array}\right) x^{i_{0}} y^{i_{1}} z^{i_{2}} D^{\left[\left(i_{1}+2 i_{2}\right) / 3\right]}
$$

et en remarquant que si $l \mid \operatorname{Dis}(Q(\Delta))$ alors $l \mid D(l \neq 3)$, on montre comme précédemment que $\varepsilon^{l} \in A^{(l)}$

On termine la démonstration en itérant le procédé et en se servant de l'inclusion $G^{\left(2^{2 \alpha}\right)} \subset G_{l^{\alpha}}$.

Fin de la preuve. Il résulte du lemme 3 l'existence d'une partition finie $\left(F_{j}\right)_{1 \leqslant j \leqslant s}$ de $E$ et d'entiers $k_{1}, \ldots, k_{s}$ tels que

$$
\forall n \in F_{j} \quad \varepsilon^{k_{j}}(n) \text { est une unité de } Z\left[\Delta_{n}\right] .
$$

Comme il n'y a qu'un nombre fini de points extrémaux formels entre 1 et $e^{k_{j}}(X)$ on en déduit, d'après le théorème 2 , que $\left|\mathscr{E}_{n}\right| \mathscr{U}_{n} \mid$ est borné indépendamment de $n$ dans $F_{j}$, et comme les $F_{j}$ sont en nombre fini on a $\overline{\lim }\left|\mathscr{E}_{n}\right| \mathscr{U}_{n} \mid<+\infty$.

Ceci termine la preuve de l'inégalité de droite de (37). 
3. Il nous reste à montrer l'inégalité

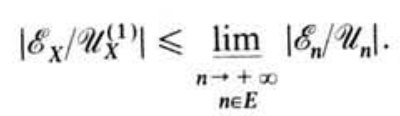

Posons $\pi=\left|\mathscr{E}_{X} / \mathscr{U}_{X}^{(1)}\right|$.

Considérons les $\pi$ premiers points extrémaux de $\mathcal{O}_{X}$ dans la région $|\varphi|_{1} \leqslant 1: \varphi_{0}=1, \varphi_{1}, \ldots, \varphi_{\pi-1}$. On a $|\varepsilon(X)|_{1}<\left|\varphi_{\pi-1}\right|_{1}<\ldots<\left|\varphi_{1}\right|_{1}<1$.

Ces points extrémaux ne sont donc pas des unités de norme 1 .

Nous allons montrer qu'ils restent non équivalents modulo $\mathscr{U}_{n}$ par spécialisation.

D'après le théorème 2 , il existe une partition finie $\left(E_{j}\right)_{1 \leqslant j \leqslant s}$ de $\tilde{Z}$ et un nombre fini $t_{j}$ de fonctions algébriques $\psi_{j}^{(i)}, 0 \leqslant i \leqslant t_{j}-1$, attachées à $E_{j}$ telles que pour tout $n$ assez grand dans $E_{j}$, la spécialisation de ces fonctions $\psi_{0}^{(j)}(n)=1, \psi_{1}^{(j)}(n), \ldots, \psi_{t_{j}-1}^{(j)}(n)$ donnent la chaîne des $t_{j}$ premières meilleures approximations de $\mathcal{O}_{n}$ de $|\cdot|_{1} \leqslant 1$ et où la dernière fonction algébrique $\psi_{t_{j}-1}^{(j)}(X)$ est équivalente à $\varphi_{\pi-1}(X)$ modulo $Q^{*}$. Enfin, pour tout $j, 1 \leqslant j \leqslant s$, il existe des entiers $i_{0}, i_{1}, \ldots, i_{n-1}$ tels que $i_{0}=1<i_{1}$ $<\ldots<i_{\pi-1}=t_{j}$ et la fonction algébrique $\psi_{i_{k}}^{(j)}$ est équivalente modulo $Q^{*}$ à $\varphi_{k}, 0 \leqslant k \leqslant \pi-1$.

On a $|\varepsilon(X)|_{1}<\left|\psi_{t_{j}-1}^{(j)}(X)\right|_{1}<\ldots<\left|\psi_{1}^{(j)}(X)\right|_{1}<1$. Ces fonctions algébriques ne sont donc pas des unités de norme 1.

Montrons que les meilleures approximations $\psi_{i_{0}}^{(j)}(n), \ldots, \psi_{i_{\pi-1}}^{(j)}(n)$ ne sont pas équivalentes modulo $\mathscr{U}_{n}$ pour tout $n$ assez grand dans $E_{j} \cap E$. Pour cela raisonnons par l'absurde.

Supposons qu'il existe $j, 1 \leqslant j \leqslant s$, et un couple $\left(i_{k}, i_{l}\right), 0 \leqslant k<l \leqslant \pi-1$, tel que $\psi_{i_{k}}^{(j)}(n)$ soit équivalente, modulo $\mathscr{U}_{n}$, à $\psi_{i_{1}}^{(j)}(n)$ pour une infinité de $n$. Comme le groupe des unités $\mathscr{U}_{n}$ opère sur les meilleures approximations, on en déduit que $\psi_{i_{1}}^{(j)} i_{k}(n)$ est une unité de $\mathcal{O}_{n}$ pour une infinité de $n$.

Comme d'autre part $\psi_{i_{L}-i_{k}}^{(i)}(X)$ est une fonction algébrique, on en déduit que c'est une unité formelle de norme 1 , ce qui est absurde.

Remarque. En fait, nous avons montré

$$
\varliminf_{\substack{n \rightarrow++\infty \\ n \in Z}}\left|\mathscr{E}_{n}\right| \mathscr{U}_{n}|\geqslant| \mathscr{E}_{X}\left|\mathscr{U}_{X}^{(1)}\right| .
$$

En effet, l'hypothèse $n \in E$ n'est pas utile dans la démonstration précédente.

\section{Exemples. 1.}

$$
\begin{gathered}
P(X)=X^{3}+4, \quad \varepsilon(X)=(-X+\Delta)^{3} / 4, \\
E_{0}=\{n \in N ; n \equiv 0(\bmod 4)\} .
\end{gathered}
$$

$\forall n \in E_{0}$, la suite des meilleures approximations de $\mathcal{O}_{n}$ est

$$
\begin{aligned}
& \varphi_{1}=-n+\Delta, \\
& \varphi_{2}=(-n+\Delta)^{2}, \\
& \varphi_{3}=(-n+\Delta)^{3} / 4, \text { unité fondamentale de } \mathcal{O}_{n} .
\end{aligned}
$$

$\varphi_{1}, \varphi_{2}, \varphi_{3}$ proviennent d'unités formelles.

$$
E_{2}=\{n \in N ; n \equiv 2(\bmod 4)\} .
$$

$\forall n \in E_{2}$, la suite des meilleures approximations de $\mathcal{O}_{n}$ est

$$
\begin{aligned}
& \varphi_{1}=-n+\Delta, \\
& \varphi_{2}=(-n+\Delta)^{2}, \\
& \varphi_{3}=n^{2} / 2+1+\left(\frac{3}{4} n^{2}-n\right) \Delta+\left(-\frac{3}{4} n+\frac{1}{2}\right) \Delta^{2}, \\
& \varphi_{4}=n^{2} / 4-1+\left(\frac{3}{4} n^{2}-n\right) \Delta+\left(\frac{3}{4} n+\frac{1}{2}\right) \Delta^{2}, \\
& \varphi_{5}=(-n+\Delta)^{3} / 2=2+\frac{3}{2} n^{2} \Delta-\frac{3}{2} n \Delta^{2}, \\
& \varphi_{6}=(-n+\Delta)^{4} / 4=-\frac{3}{4} n^{4}-4 n+\left(-\frac{3}{4} n^{2}+1\right) \Delta+\frac{3}{2} n^{2} \Delta^{2}, \\
& \varphi_{7}=(-n+\Delta)^{5} / 4, \\
& \varphi_{8}=(-n+\Delta)^{6} / 4^{2}, \quad \text { unité fondamentale de } \mathcal{O}_{n} .
\end{aligned}
$$

$\varphi_{1}, \varphi_{2}, \varphi_{5}, \varphi_{6}, \varphi_{7}, \varphi_{8}$ proviennent d'unités formelles mais $\varphi_{3}, \varphi_{4}$ proviennent de faces.

$$
\left|\mathscr{E}_{X}\right| \mathscr{U}_{X}^{(1)} \mid=3, \quad E=E_{0} \cup E_{2}=\left\{n ; \varepsilon(n) \text { entier de } K_{n}\right\}, \quad \varlimsup_{\substack{n \in E \\ n \rightarrow+\infty}}\left|\mathscr{E}_{n}\right| \mathscr{U}_{n} \mid=8 .
$$

2.

$$
\begin{gathered}
P(X)=X^{3}-9 X+9, \quad \varepsilon(X)=-X^{3} / 3+3 X-4+\left(X^{2} / 3+X-2\right) \Delta-\Delta^{2}, \\
E_{0}=\{n \in N, n \equiv 0(\bmod 3)\} .
\end{gathered}
$$

$\forall n \geqslant 12$, la suite des meilleures approximations de $\mathcal{O}_{n}$ est

$$
\begin{aligned}
& \varphi_{1}=-n+1+\Delta, \\
& \varphi_{2}=n-\Delta, \\
& \varphi_{3}=-n^{2} / 3+1+n \Delta / 3, \\
& \varphi_{4}=-n-3+(-n+1) \Delta+\Delta^{2}, \\
& \varphi_{5}=-n^{2}-3 n+9+(-n+3) \Delta+2 \Delta^{2}, \\
& \varphi_{6}=-n^{3} / 3+4 n-5+(n-2) \Delta+(n / 3-1) \Delta^{2}, \\
& \varphi_{7}=-n^{3} / 3+3 n-4+\left(n^{2} / 3+n-2\right) \Delta-\Delta^{2},
\end{aligned}
$$

$\varphi_{7}$ unité fondamentale de $\mathcal{O}_{n}$. 
$\varphi_{1}, \varphi_{3}, \varphi_{4}, \varphi_{6}$ proviennent de faces, $\varphi_{2}, \varphi_{5}$ proviennent de points extrémaux, $\varphi_{7}$ provient d'une unité formelle.

$$
\left|\mathscr{E}_{X} / \mathscr{U}_{X}^{(1)}\right|=3, \quad E=E_{0}=\left\{n ; \varepsilon(n) \text { entier de } K_{n}\right\}, \quad \varlimsup_{\substack{n \in E \\ n \rightarrow+\infty}}\left|\mathscr{E}_{n}\right| \mathscr{U}_{n} \mid=7 .
$$

Remarque sur le théorème 1 . Pour montrer le théorème 1 , on pourrait penser utiliser le résultat de $\mathrm{H}$. C. Williams [6] qui dit intuitivement la chose suivante:

"Si $\varphi$ est un élément d'un ordre $\mathcal{O}$ de petite norme alors $\varphi$ est une meilleure approximation de $G$ ". Précisément:

Soit $\mathcal{O}=\boldsymbol{Z}+\boldsymbol{Z} \mu+\boldsymbol{Z} v$ un ordre de $K$ (corps cubique pur) et $D_{0}$ son discriminant. Soit $\varphi$ un élément non nul de $\mathcal{O}$. Si:

$$
|N(\varphi)|<\sqrt[4]{D_{0} / 27}
$$

alors $\varphi$ est une meilleure approximation de $\mathcal{O}$.

Malheureusement, l'approximation formelle obtenue de façon générale et le résultat de H.C.Williams ne sont pas suffisants pour démontrer le théorème 1 En effet, si $\varphi$ est un point extrémal de $\mathcal{O}_{X}$ on a:

$\operatorname{deg} N(\varphi) \leqslant 3 d-2$ (voir proposition 3) et $\operatorname{deg}(\operatorname{Disc} \Delta)=6 d$,

l'inégalité (40) n'est donc pas satisfaite asymptotiquement.

\section{Bibliographie}

[1] E. Dubois et R. Paysant-Le R oux, Sur la longueur du développement en fraction continue de $\sqrt{f(n)}$, Journées Arithmétiques, Marseille 89

2] Y. Hellatilan et R. Paysant-Le Roux, Unités de certains sous-anneaux des corps de fonctions algébriques, Acta Arith. 48 (1987), 9-47.

[3] M. Neubrand, Einheiten in algebraischen Funktionen- und Zahlkörpern, J. Reine Angew. Math. 303/304 (1978), 170-203.

[4] R. Paysant-Le Roux, Calibre d'un corps global, J. Number Theory 30 (1988), 267-287.

[5]

6 (1961), 393-413; 7 (1962), 287-298.

6] H. C. Williams, The period length of Voronoi's algorithm for certain cubic orders, Publ. Math. Debrecen, $\mathrm{N}^{\circ}$ 3-4, 37; 91 (1990), 05, 245-265.

\section{R. Paysant-Le Roux}

M. L. Gaune

UNIVERSITÉ DE CAEN

14032 Caen Cedex, France

E. Dubois

ISMRA ET UNIVERSITÉ

14032 Caen Cedex, France

\section{A Dirichlet series for modular forms of degree $n$}

by

\section{Aloys KRIEG (Münster)}

1. Introduction. In a recent paper Kohnen and Skoruppa [6] introduced a new type of Dirichlet series, which is associated with the Fourier-Jacobi expansion of a pair $f, g$ of Siegel cusp forms of the same weight and degree 2. The proof is based on the Rankin-Selberg method. If $f=g$ is a simultaneous Hecke eigenform in the so-called Maß Spezialschar, this Dirichlet series is proportional to the spinor zeta function attached to $f$ by Andrianov.

Then Yamazaki [17], [18] derived the analytic continuation and functional equation for this type of Dirichlet series in the case of arbitrary degree $n$. The main point of his proof is the analytic continuation of an Eisenstein series of Klingen type, which is derived from Langlands' theory. Recently Raghavan and Sengupta [13] obtained analogous results for Hermitian modular forms of degree 2 with respect to the Gaussian number field.

In this paper we give an elementary proof of Yamazaki's result [17], which only makes use of well-known properties of Epstein zeta functions. A modification of the arguments and another application of the Rankin-Selberg method lead to the corresponding result for Hermitian modular forms of degree $n$ with respect to the Gaussian number field. Finally, we deal with modular forms of quaternions of degree $n$ (cf. [11], [7]). We derive analytic continuation and functional equation of the attached Dirichlet series. If $f$ and $g$ belong to the Maaß space (cf. [8]) and if $f$ is a simultaneous eigenform under all Hecke operators, then the Dirichlet series possesses an Euler product expansion and is proportional to the Andrianov zeta function attached to $f$.

2. Preliminaries. A good deal of work can be done for all the three types of modular forms simultaneously just as in [7]. Therefore let $\boldsymbol{F}$ stand for the field $\boldsymbol{R}$ of real numbers resp. $\boldsymbol{C}$ of complex numbers resp. for the skew-field $\boldsymbol{H}$ of Hamiltonian quaternions. Set $r=[\boldsymbol{F}: \boldsymbol{R}]$ and denote the standard basis of $\boldsymbol{F}$ over $\boldsymbol{R}$, by $e_{1}, \ldots, e_{r}$, where

$$
e_{1}=1, \quad e_{2}^{2}=e_{3}^{2}=e_{4}^{2}=-1, \quad e_{2} e_{3}=-e_{3} e_{2}=e_{4} .
$$

Let $a \mapsto \bar{a}$ be the canonical involution on $\boldsymbol{F}$ and define

$$
\operatorname{Re}(a):=\frac{1}{2}(a+\bar{a}), \quad\langle a, b\rangle:=\operatorname{Re}(\bar{a} b), \quad N(a):=a \bar{a} .
$$

